\title{
Automatic Verification of Integer Array Programs ${ }^{\star}$
}

\author{
Marius Bozga ${ }^{1}$, Peter Habermehl ${ }^{2}$, Radu Iosif $^{1}$, Filip Konečný ${ }^{1,3}$, and Tomáš Vojnar $^{3}$ \\ ${ }^{1}$ VERIMAG, CNRS, 2 av. de Vignate, 38610 Gières, France \\ \{bozga, iosif\}@imag.fr \\ 2 LIAFA, University Paris 7, Case 7014, 75205 Paris 13, France \\ haberm@liafa.jussieu.fr \\ ${ }^{3}$ FIT BUT, Božetěchova 2, 61266, Brno, Czech Republic \\ \{ikonecny, vojnar\}@fit.vutbr.cz
}

\begin{abstract}
We provide a verification technique for a class of programs working on integer arrays of finite, but not a priori bounded length. We use the logic of integer arrays SIL [13] to specify pre- and post-conditions of programs and their parts. Effects of non-looping parts of code are computed syntactically on the level of SIL. Loop pre-conditions derived during the computation in SIL are converted into counter automata (CA). Loops are automatically translatedpurely on the syntactical level-to transducers. Pre-condition CA and transducers are composed, and the composition over-approximated by flat automata with difference bound constraints, which are next converted back into SIL formulae, thus inferring post-conditions of the loops. Finally, validity of post-conditions specified by the user in SIL may be checked as entailment is decidable for SIL.
\end{abstract}

\section{Introduction}

Arrays are an important data structure in all common programming languages. Automatic verification of programs using arrays is a difficult task since they are of a finite, but often not a priori fixed length, and, moreover, their contents may be unbounded too. Nevertheless, various approaches for automatic verification of programs with arrays have recently been proposed.

In this paper, we consider programs over integer arrays with assignments, conditional statements, and non-nested while loops. Our verification technique is based on a combination of the logic of integer arrays SIL [13], used for expressing pre-/post-conditions of programs and their parts, and counter automata (CA) and transducers, into which we translate both SIL formulae and program loops in order to be able to compute the effect of loops and to be able to check entailment.

SIL (Single Index Logic) allows one to describe properties over arrays of integers and scalar variables. SIL uses difference bound constraints to compare array elements situated within a window of a constant size. For instance, the formula $(\forall i .0 \leq i \leq$ $\left.n_{1}-1 \rightarrow b[i] \geq 0\right) \wedge\left(\forall i .0 \leq i \leq n_{2}-1 \rightarrow c[i]<0\right)$ describes a post-condition of a program partitioning an array $a$ into an array $b$ containing its positive elements and an array $c$ containing its negative elements. SIL formulae are interpreted over program

\footnotetext{
* This work was supported by the French project RNTL AVERILES, the Czech Science Foundation (projects 102/07/0322, 102/09/H042), the Barrande project MEB 020840, and the Czech Ministry of Education by the project MSM 0021630528.
} 
states assigning integers to scalar variables and finite sequences of integers to array variables. As already proved in [13], the set of models of an $\exists^{*} \forall^{*}$-SIL formula corresponds naturally to the set of traces of a flat CA with loops labelled by difference bound constraints. This entails decidability of the satisfiability problem for $\exists * \forall^{*}$-SIL.

In this paper we take a novel perspective on the connection between $\exists^{*} \forall^{*}$-SIL and CA, allowing to benefit from the advantages of both formalisms. Indeed, the logic is useful to express human-readable pre-/post-conditions of programs and their parts, and to compute the post-image of (non-looping) program statements symbolically. On the other hand, automata are suitable for expressing the effects of program loops.

In particular, given an $\exists^{*} \forall^{*}$-SIL formula, we can easily compute the strongest postcondition of an assignment or a conditional statement in the same fragment of the logic. Upon reaching a program loop, we then translate the $\exists^{*} \forall^{*}$-SIL formula $\varphi$ describing the set of states at the beginning of the loop into a CA $A_{\varphi}$ encoding its set of models. Next, to characterise the effect of a loop $L$, we translate it—purely syntactically-into a transducer $T_{L}$, i.e., a CA describing the input/output relation on scalars and array elements implemented by $L$. The post-condition of $L$ is then obtained by composing $T_{L}$ with $A_{\varphi}$. The result of the composition is a CA $B_{\varphi, L}$ representing the exact set of states after any number of iterations of $L$. Finally, we translate $B_{\varphi, L}$ back into $\exists^{*} \forall^{*}$-SIL, obtaining a post-condition of $L$ w.r.t. $\varphi$. However, due to the fact that counter automata are more expressive than $\exists^{*} \forall^{*}$-SIL, this final step involves a (refinable) abstraction. We first generate a flat CA that over-approximates the set of traces of $B_{\varphi, L}$, and then translate the flat CA back into $\exists^{*} \forall^{*}$-SIL.

Our approach thus generates automatically a human-readable post-condition for each program loop, giving the end-user some insight of what the program is doing. Moreover, as these post-conditions are expressed in a decidable logic, they can be used to check entailment of user-specified post-conditions given in the same logic.

We validate our approach by successfully and fully algorithmically verifying several array-manipulating programs, like splitting of an array into positive and negative elements, rotating an array, inserting into a sorted array, etc. Some of the steps were done manually as we have not yet implemented all of the techniques-a full implementation that will allow us to do more examples is underway.

Due to space reasons, we skip below some details of the techniques and their proofs, which are deferred to [4].

Related Work. The area of automated verification of programs with arrays and/or synthesising loop invariants for such programs has recently received a lot of attention. For

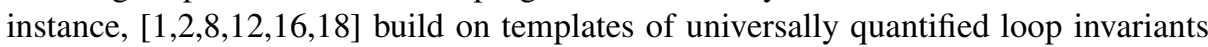
and/or atomic predicates provided by the user. The form of the sought invariants is then based on these templates. Inferring the invariants is tackled by various approaches, such as predicate abstraction using predicates with Skolem constants [8], constraint-based invariant synthesis [12], or predicate abstraction combined with interpolation-based refinement [16].

In [20], an interpolating saturation prover is used for deriving invariants from finite unfoldings of loops. In the very recent work of [17], loop invariants are synthesised by first deriving scalar invariants, combining them with various predefined first-order array axioms, and finally using a saturation prover for generating the loop invariants on arrays. 
This approach can generate invariants containing quantifier alternation. A disadvantage is that, unlike our approach, the method does not take into account loop preconditions, which are sometimes necessary to find reasonable invariants. Also, the method does not generate invariants in a decidable logical fragment, in general.

Another approach, based on abstract interpretation, was used in [11]. Here, arrays are suitably partitioned, and summary properties of the array segments are tracked. The partitioning is based on heuristics related to tracking the position of index variables. These heuristics, however, sometimes fail, and human guidance is needed. The approach was recently improved in [15] by using better partitioning heuristics and relational abstract domains to keep track of the relations of the particular array slices.

Recently, several works have proposed decidable logics capable of expressing complex properties of arrays [36 3 10 21$]$. In general, these logics lack the capability of universally relating two successive elements of arrays, which is allowed in our previous work [13 14]. Moreover, the logics of [3|6 9 10]21] do not give direct means of automatically dealing with program loops, and hence, verifying programs with arrays. In this work, we provide a fully algorithmic verification technique that uses the decidable logic of [13]. Unlike many other works, we do not synthesise loop invariants, but directly post-conditions of loops with respect to given preconditions, using a two-way automata-logic connection that we establish.

\section{Preliminaries}

For a set $A$, we denote by $A^{*}$ the set of finite sequences of elements from $A$. For such a sequence $\sigma \in A^{*}$, we denote by $|\sigma|$ its length, and by $\sigma_{i}$ the element at position $i$, for $0 \leq i<|\sigma|$. We denote by $\mathbb{N}$ the set of natural numbers, and by $\mathbb{Z}$ the set of integers. For a function $f: A \rightarrow B$ and a set $S \subseteq A$, we denote by $f \downarrow_{S}$ the restriction of $f$ to $S$. This notation is naturally lifted to sets, pairs or sequences of functions.

Given a formula $\varphi$, we denote by $F V(\varphi)$ the set of its free variables. If we denote a formula as $\varphi\left(x_{1}, \ldots, x_{n}\right)$, we assume $F V(\varphi) \subseteq\left\{x_{1}, \ldots, x_{n}\right\}$. For $\varphi\left(x_{1}, \ldots, x_{n}\right)$, we denote by $\varphi\left[t_{1} / x_{1}, \ldots, t_{n} / x_{n}\right]$ the formula which is obtained from $\varphi$ by replacing each free occurrence of $x_{1}, \ldots, x_{n}$ by the terms $t_{1}, \ldots, t_{n}$, respectively. Moreover, we denote by $\varphi\left[t / x_{1}, \ldots, x_{n}\right]$ the formula that arises from $\varphi$ when all free occurrences of all the variables $x_{1}, \ldots, x_{n}$ are replaced by the same term $t$. Given a formula $\varphi$ and a valuation $v$ of its free variables, we write $\nu \models \varphi$ if by replacing each free variable $x$ of $\varphi$ with $v(x)$ we obtain a valid formula. By $\models \varphi$ we denote the fact that $\varphi$ is valid.

A difference bound constraint (DBC) is a conjunction of inequalities of the forms (1) $x-y \leq c$, (2) $x \leq c$, or (3) $x \geq c$, where $c \in \mathbb{Z}$ is a constant. We denote by $\top$ (true) the empty DBC.

A counter automaton (CA) is a tuple $A=\langle X, Q, I, \rightarrow, F\rangle$, where: $X$ is a finite set of counters ranging over $\mathbb{Z}, Q$ is a finite set of control states, $I \subseteq Q$ is a set of initial states, $\rightarrow$ is a transition relation given by a set of rules $q \stackrel{\varphi\left(X, X^{\prime}\right)}{\longrightarrow} q^{\prime}$ where $\varphi$ is an arithmetic formula relating current values of counters $X$ to their future values $X^{\prime}=\left\{x^{\prime} \mid x \in X\right\}$, and $F \subseteq Q$ is a set of final states. 
A configuration of a CA $A$ is a pair $\langle q, v\rangle$ where $q \in Q$ is a control state, and $v: X \rightarrow \mathbb{Z}$ is a valuation of the counters in $X$. For a configuration $c=\langle q, v\rangle$, we designate by $\operatorname{val}(c)=v$ the valuation of the counters in $c$. A configuration $\left\langle q^{\prime}, v^{\prime}\right\rangle$ is an immediate successor of $\langle q, v\rangle$ if and only if $A$ has a transition rule $q \stackrel{\varphi\left(X, X^{\prime}\right)}{\longrightarrow} q^{\prime}$ such that $v \cup v^{\prime} \models$ $\varphi$. Given two control states $q, q^{\prime} \in Q$, a run of $A$ from $q$ to $q^{\prime}$ is a finite sequence of configurations $c_{1} c_{2} \ldots c_{n}$ with $c_{1}=\langle q, v\rangle, c_{n}=\left\langle q^{\prime}, v^{\prime}\right\rangle$ for some valuations $v, v^{\prime}: X \rightarrow \mathbb{Z}$, and $c_{i+1}$ is an immediate successor of $c_{i}$, for all $1 \leq i<n$. Let $\mathcal{R}(A)$ denote the set of runs of $A$ from some initial state $q_{0} \in I$ to some final state $q_{f} \in F$, and $\operatorname{Tr}(A)=$ $\left\{\operatorname{val}\left(c_{1}\right) \operatorname{val}\left(c_{2}\right) \ldots \operatorname{val}\left(c_{n}\right) \mid c_{1} c_{2} \ldots c_{n} \in \mathcal{R}(A)\right\}$ be its set of traces.

For two counter automata $A_{i}=\left\langle X_{i}, Q_{i}, I_{i}, \rightarrow_{i}, F_{i}\right\rangle, i=1,2$ we define the product automaton as $A_{1} \otimes A_{2}=\left\langle X_{1} \cup X_{2}, Q_{1} \times Q_{2}, I_{1} \times I_{2}, \rightarrow, F_{1} \times F_{2}\right\rangle$, where $\left\langle q_{1}, q_{2}\right\rangle \stackrel{\varphi}{\rightarrow}\left\langle q_{1}^{\prime}, q_{2}^{\prime}\right\rangle$ if and only if $q_{1} \stackrel{\varphi_{1}}{\rightarrow}{ }_{1} q_{1}^{\prime}, q_{2} \stackrel{\varphi_{2}}{\rightarrow} q_{2}^{\prime}$ and $\models \varphi \leftrightarrow \varphi_{1} \wedge \varphi_{2}$. We have that, for all sequences $\sigma \in \operatorname{Tr}\left(A_{1} \otimes A_{2}\right), \sigma \downarrow_{X_{1}} \in \operatorname{Tr}\left(A_{1}\right)$ and $\sigma \downarrow_{X_{2}} \in \operatorname{Tr}\left(A_{2}\right)$, and vice versa.

\section{Counter Automata as Recognisers of States and Transitions}

In the rest of this section, let $\mathbf{a}=\left\{a_{1}, a_{2}, \ldots, a_{k}\right\}$ be a set of array variables, and $\mathbf{b}=$ $\left\{b_{1}, b_{2}, \ldots, b_{m}\right\}$ be a set of scalar variables. A state $\langle\alpha, \mathbf{l}\rangle$ is a pair of valuations $\alpha: \mathbf{a} \rightarrow$ $\mathbb{Z}^{*}$, and $\mathbf{\imath}: \mathbf{b} \rightarrow \mathbb{Z}$. For simplicity, we assume that $\left|\alpha\left(a_{1}\right)\right|=\left|\alpha\left(a_{2}\right)\right|=\ldots=\left|\alpha\left(a_{k}\right)\right|>0$, and denote by $|\alpha|$ the size of the arrays in the state.

In the following, let $X$ be a set of counters that is partitioned into value counters $\mathbf{x}=\left\{x_{1}, x_{2}, \ldots, x_{k}\right\}$, index counters $\mathbf{i}=\left\{i_{1}, i_{2}, \ldots, i_{k}\right\}$, parameters $\mathbf{p}=\left\{p_{1}, p_{2}, \ldots, p_{m}\right\}$, and working counters $\mathbf{w}$. Notice that $\mathbf{a}$ is in a 1:1 correspondence with both $\mathbf{x}$ and $\mathbf{i}$, and that $\mathbf{b}$ is in a 1:1 correspondence with $\mathbf{p}$.

Definition 1. Let $\langle\alpha, \mathrm{l}\rangle$ be a state. A sequence $\sigma \in(X \rightarrow \mathbb{Z})^{*}$ is said to be consistent with $\langle\alpha, \mathbf{\imath}\rangle$, denoted $\sigma \vdash\langle\alpha, \mathbf{l}\rangle$ if and only if, for all $1 \leq p \leq k$, and all $1 \leq r \leq m$ :

1. for all $q \in \mathbb{N}$ with $0 \leq q<|\sigma|$, we have $0 \leq \sigma_{q}\left(i_{p}\right) \leq|\alpha|$,

2. for all $q, r \in \mathbb{N}$ with $0 \leq q<r<|\sigma|$, we have $\sigma_{q}\left(i_{p}\right) \leq \sigma_{r}\left(i_{p}\right)$,

3. for all $s \in \mathbb{N}$ with $0 \leq s \leq|\alpha|$, there exists $0 \leq q<|\sigma|$ such that $\sigma_{q}\left(i_{p}\right)=s$,

4. for all $q \in \mathbb{N}$ with $0 \leq q<|\sigma|$, if $\sigma_{q}\left(i_{p}\right)=s<|\alpha|$, then $\sigma_{q}\left(x_{p}\right)=\alpha\left(a_{p}\right)_{s}$,

5. for all $q \in \mathbb{N}$ with $0 \leq q<|\sigma|$, we have $\sigma_{q}\left(p_{r}\right)=\mathfrak{\imath}\left(b_{r}\right)$.

Intuitively, a run of a CA represents the contents of a single array by traversing all of its entries in one move from the left to the right. The contents of multiple arrays is represented by arbitrarily interleaving the traversals of the different arrays. From this point of view, for a run to correspond to some state (i.e., to be consistent with it), it must be the case that each index counter either keeps its value or grows at each step of the run (point 2 of Def. 1) while visiting each entry within the array (points 1 and 3 of Def. 11 1 The value of a certain entry of an array $a_{p}$ is coded by the value that

\footnotetext{
${ }^{1}$ In fact, each index counter reaches the value $|\alpha|$ which is by one more than what is needed to traverse an array with entries $0, \ldots,|\alpha|-1$. The reason is technical, related to the composition with transducers representing program loops (which produce array entries with a delay of one step and hence need the extra index value to produce the last array entry) as will become clear later. Note that the entry at position $|\alpha|$ is left unconstrained.
} 
the array counter $x_{p}$ has when the index counter $i_{p}$ contains the position of the given entry (point 4 of Def.11). Finally, values of scalar variables are encoded by values of the appropriate parameter counters which stay constant within a run (point 5 of Def. 1).

A CA is said to be state consistent if and only if for every trace $\sigma \in \operatorname{Tr}(A)$, there exists a (unique) state $\langle\alpha, \mathrm{l}\rangle$ such that $\sigma \vdash\langle\alpha, \mathrm{l}\rangle$. We denote $\Sigma(A)=\{\langle\alpha, \mathrm{l}\rangle \mid \exists \sigma \in$ $\operatorname{Tr}(A) . \sigma \vdash\langle\alpha, \mathrm{l}\rangle\}$ the set of states recognised by a CA.

A consequence of Definition 1 is that, in between two adjacent positions of a trace, in a state-consistent $\mathrm{CA}$, the index counters never increase by more than one. Consequently, each transition whose relation is non-deterministic w.r.t. an index counter can be split into two transitions: an idle (no change) and a tick (increment by one). In the following, we will silently assume that each transition of a state-consistent CA is either idle or tick w.r.t. a given index counter.

For any set $U=\left\{u_{1}, \ldots, u_{n}\right\}$, let us denote $U^{i}=\left\{u_{1}^{i}, \ldots, u_{n}^{i}\right\}$ and $U^{o}=\left\{u_{1}^{o}, \ldots, u_{n}^{o}\right\}$. If $s=\langle\alpha, \mathrm{l}\rangle$ and $t=\langle\beta, \kappa\rangle$ are two states such that $|\alpha|=|\beta|$, the pair $\langle s, t\rangle$ is referred to as a transition. A CA $T=\langle X, Q, I, \rightarrow, F\rangle$ is said to be a transducer iff its set of counters $X$ is partitioned into: input counters $\mathbf{x}^{i}$ and output counters $\mathbf{x}^{o}$, where $\mathbf{x}=\left\{x_{1}, x_{2}, \ldots, x_{k}\right\}$, index counters $\mathbf{i}=\left\{i_{1}, i_{2}, \ldots, i_{k}\right\}$, input parameters $\mathbf{p}^{i}$ and output parameters $\mathbf{p}^{o}$, where $\mathbf{p}=\left\{p_{1}, p_{2}, \ldots, p_{m}\right\}$, and working counters $\mathbf{w}$.

Definition 2. A sequence $\sigma \in(X \rightarrow \mathbb{Z})^{*}$ is said to be consistent with a transition $\langle s, t\rangle$, where $s=\langle\alpha, \mathrm{l}\rangle$ and $t=\langle\beta, \kappa\rangle$, denoted $\sigma \vdash\langle s, t\rangle$ if and only if, for all $1 \leq p \leq k$ and all $1 \leq r \leq m$ :

1. for all $q \in \mathbb{N}$ with $0 \leq q<|\sigma|$, we have $0 \leq \sigma_{q}\left(i_{p}\right) \leq|\alpha|$,

2. for all $q, r \in \mathbb{N}$ with $0 \leq q<r<|\sigma|$, we have $\sigma_{q}\left(i_{p}\right) \leq \sigma_{r}\left(i_{p}\right)$,

3. for all $s \in \mathbb{N}$ with $0 \leq s \leq|\alpha|$, there exists $0 \leq q<|\sigma|$ such that $\sigma_{q}\left(i_{p}\right)=s$,

4. for all $q \in \mathbb{N}$ with $0 \leq q<|\sigma|$, if $\sigma_{q}\left(i_{p}\right)=s<|\alpha|$, then $\sigma_{q}\left(x_{p}^{i}\right)=\alpha\left(a_{p}\right)_{s}$,

5. for all $q \in \mathbb{N}$ with $0 \leq q<|\sigma|$, if $\sigma_{q}\left(i_{p}\right)=s>0$, then $\sigma_{q}\left(x_{p}^{o}\right)=\beta\left(a_{p}\right)_{s-1}$,

6. for all $q \in \mathbb{N}$ with $0 \leq q<|\sigma|$, we have $\sigma_{q}\left(p_{r}^{i}\right)=\mathfrak{\imath}\left(b_{r}\right)$ and $\sigma\left(p_{r}^{o}\right)=\kappa\left(b_{r}\right)$.

The intuition behind the way the transducers represent transitions of programs with arrays is very similar to the way we use counter automata to represent states of such programs - the transducers just have input as well as output counters whose values in runs describe the corresponding input and output states. Note that the definition of transducers is such that the output values occur with a delay of exactly one step w.r.t. the corresponding input (cf. point 5 in Def. 2) 2

A transducer $T$ is said to be transition consistent iff for every trace $\sigma \in \operatorname{Tr}(T)$ there exists a transition $\langle s, t\rangle$ such that $\sigma \vdash\langle s, t\rangle$. We denote $\Theta(T)=\{\langle s, t\rangle \mid \exists \sigma \in \operatorname{Tr}(T) . \sigma \vdash$ $\langle s, t\rangle\}$ the set of transitions recognised by a transducer.

Dependencies between Index Counters. Counter automata and transducers can represent one array in more than one way, which poses problems when composing them. For instance, the array $a=\{0 \mapsto 4,1 \mapsto 3,2 \mapsto 2\}$ may be encoded, e.g., by the runs

\footnotetext{
${ }^{2}$ The intuition is that it takes the transducer one step to compute the output value, once it reads the input. It is possible to define a completely synchronous transducer, we, however, prefer this definition for technical reasons related to the translation of program loops into transducers.
} 
$(0,4),(0,4),(1,3),(2,2),(2,2)$ and $(0,4),(1,3),(1,3),(2,2)$, where the first elements of the pairs are the values taken by the index counters, and the second elements are the values taken by the value counters corresponding to $a$. To obtain a sufficient criterion that guarantees that a CA and a transducer can be composed, meaning that they share a common representation of arrays, we introduce a notion of dependence. Intuitively, we call two or more index counters dependent if they increase at the same moments in all possible runs of a $\mathrm{CA}$ or transducer.

For the rest of this section, let $X \subset \mathbf{i}$ be a fixed set of index counters. A dependency $\delta$ is a conjunction of equalities between elements belonging $X$. For a sequence of valuations $\sigma \in(X \rightarrow \mathbb{Z})^{*}$, we denote $\sigma \models \delta$ if and only if $\sigma_{l}=\delta$, for all $0 \leq l<|\sigma|$.

For a dependency $\delta$, we denote $[[\delta]]=\left\{\sigma \in(X \rightarrow \mathbb{Z})^{*} \mid\right.$ there exists a state $s$ such that $\sigma \vdash s$ and $\sigma \models \delta\}$, i.e., the set of all sequences that correspond to an array and that satisfy $\delta$. A dependency $\delta_{1}$ is said to be stronger than another dependency $\delta_{2}$, denoted $\delta_{1} \rightarrow \delta_{2}$, if and only if the first order logic entailment between $\delta_{1}$ and $\delta_{2}$ is valid. Note that $\delta_{1} \rightarrow \delta_{2}$ if and only if $\left[\left[\delta_{1}\right]\right] \subseteq\left[\left[\delta_{2}\right]\right]$. If $\delta_{1} \rightarrow \delta_{2}$ and $\delta_{2} \rightarrow \delta_{1}$, we write $\delta_{1} \leftrightarrow \delta_{2}$. For a state consistent counter automaton (transition consistent transducer) $A$, we denote by $\Delta(A)$ the strongest dependency $\delta$ such that $\operatorname{Tr}(A) \subseteq[[\delta]$.

Definition 3. A $C A A=\langle\mathbf{x}, Q, I, \rightarrow, F\rangle$, where $\mathbf{x} \subseteq X$, is said to be state-complete if and only if for all states $s \in \Sigma(A)$, and each sequence $\sigma \in(X \rightarrow \mathbb{Z})^{*}$, such that $\sigma \vdash s$ and $\sigma \models \Delta(A)$, we have $\sigma \in \operatorname{Tr}(A)$.

Intuitively, an automaton $A$ is state-complete if it represents any state $s \in \Sigma(A)$ in all possible ways w.r.t. the strongest dependency relation on its index counters.

Composing Counter Automata with Transducers. For a counter automaton $A$ and a transducer $T, \Sigma(A)$ represents a set of states, whereas $\Theta(T)$ is a transition relation. A natural question is whether the post-image of $\Sigma(A)$ via the relation $\Theta(T)$ can be represented by a $\mathrm{CA}$, and whether this automaton can be effectively built from $A$ and $T$.

Theorem 1. If A is a state-consistent and state-complete counter automaton with value counters $\mathbf{x}=\left\{x_{1}, \ldots, x_{k}\right\}$, index counters $\mathbf{i}=\left\{i_{1}, \ldots, i_{k}\right\}$, and parameters $\mathbf{p}=\left\{p_{1}, \ldots, p_{m}\right\}$, and $T$ is a transducer with input (output) counters $\mathbf{x}^{i}\left(\mathbf{x}^{o}\right)$, index counters $\mathbf{i}$, and input (output) parameters $\mathbf{p}^{i}\left(\mathbf{p}^{o}\right)$ such that $\Delta(T)\left[\mathbf{x} / \mathbf{x}^{i}\right] \rightarrow \Delta(A)$, then one can build a stateconsistent counter automaton $B$, such that $\Sigma(B)=\{t \mid \exists s \in \Sigma(A) .\langle s, t\rangle \in \Theta(T)\}$, and, moreover $\Delta(B) \rightarrow \Delta(T)\left[\mathbf{x} / \mathbf{x}^{i}\right]$.

\section{Singly Indexed Logic}

We consider three types of variables. The scalar variables $b, b_{1}, b_{2}, \ldots \in B$ Var appear in the bounds that define the intervals in which some array property is required to hold and within constraints on non-array data variables. The index variables $i, i_{1}, i_{2}, \ldots \in I$ Var and array variables $a, a_{1}, a_{2}, \ldots \in A$ Var are used in array terms. The sets $B V a r, I V a r$, and AVar are assumed to be pairwise disjoint.

Fig. 1 shows the syntax of the Single Index Logic SIL. We use the symbol $\top$ to denote the boolean value true. In the following, we will write $i<f$ instead of $i \leq f-1$, 


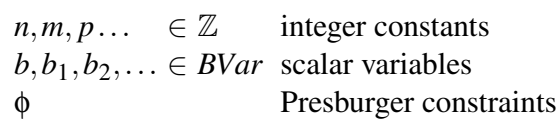

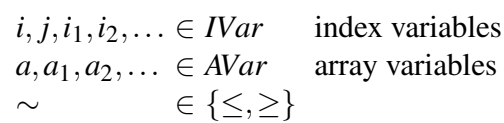

array-bound terms guard expressions value expressions formulae

Fig. 1. Syntax of the Single Index Logic

$i=f$ instead of $f \leq i \leq f, \varphi_{1} \vee \varphi_{2}$ instead of $\neg\left(\neg \varphi_{1} \wedge \neg \varphi_{2}\right)$, and $\forall i . v(i)$ instead of $\forall i$. $\top \rightarrow \mathrm{v}(i)$. If $B_{1}\left(b_{1}\right), \ldots, B_{n}\left(b_{n}\right)$ are bound terms with free variables $b_{1}, \ldots, b_{n} \in$ $B V a r$, respectively, we write any Presburger formula $\varphi$ on terms $a_{1}\left[B_{1}\right], \ldots, a_{n}\left[B_{n}\right]$ as a shorthand for $\left(\bigwedge_{k=1}^{n} \forall j \cdot j=B_{k} \rightarrow a_{k}[j]=b_{k}^{\prime}\right) \wedge \varphi\left[b_{1}^{\prime} / a_{1}\left[B_{1}\right], \ldots, b_{n}^{\prime} / a_{n}\left[B_{n}\right]\right]$, where $b_{1}^{\prime}, \ldots, b_{n}^{\prime}$ are fresh scalar variables.

The semantics of a formula $\varphi$ is defined in terms of the forcing relation $\langle\alpha, \mathbf{l}\rangle \models \varphi$ between states and formulae. In particular, $\langle\alpha, \mathbf{l}\rangle \models \forall i . \gamma(i, \mathbf{b}) \rightarrow v(i, \mathbf{a}, \mathbf{b})$ if and only if, for all values $n$ in the set $\bigcap\{[-m,|\alpha|-m-1] \mid a[i+m]$ occurs in $v\}$, if $\mathbf{\imath}=\gamma[n / i]$, then also $\imath \cup \alpha=v[n / i]$. Intuitively, the value expression $\gamma$ should hold only for those indices that do not generate out of bounds array references.

We denote $[[\varphi]]=\{\langle\alpha, \mathrm{l}\rangle|\langle\alpha, \mathrm{l}\rangle|=\varphi\}$. The satisfiability problem asks, for a given formula $\varphi$, whether $[[\varphi]] \stackrel{?}{=} \emptyset$. We say that an automaton $A$ and a SIL formula $\varphi$ correspond if and only if $\Sigma(A)=[[\varphi]]$.

The $\exists^{*} \forall^{*}$ fragment of SIL is the set of SIL formulae which, when written in prenex normal form, have the quantifier prefix of the form $\exists i_{1} \ldots \exists i_{n} \forall j_{1} \ldots \forall j_{m}$. As shown in [13] (for a slightly more complex syntax), the $\exists^{*} \forall^{*}$ fragment of SIL is equivalent to the set of existentially quantified boolean combinations of (1) Presburger constraints on scalar variables $\mathbf{b}$, and (2) array properties of the form $\forall i . \gamma(i, \mathbf{b}) \rightarrow v(i, \mathbf{b}, \mathbf{a})$.

Theorem 2 ([13]). The satisfiability problem is decidable for the $\exists^{*} \forall^{*}$ fragment of SIL.

Below, we establish a two-way connection between $\exists^{*} \forall^{*}$-SIL and counter automata. Namely, we show how loop pre-conditions written in $\exists^{*} \forall^{*}$-SIL can be translated to CA in a way suitable for their further composition with transducers representing program loops (for this reason the translation differs from [13]). Then, we show how $\exists^{*} \forall^{*}$-SIL formulae can be derived from the CA that we obtain as the product of loop transducers and pre-condition $\mathrm{CA}$.

\subsection{From $\exists^{*} \forall^{*}$-SIL to Counter Automata}

Given a pre-condition $\varphi$ expressed in $\exists^{*} \forall^{*}$-SIL, we build a corresponding counter automaton $A$, i.e., $\Sigma(A)=[[\varphi]]$. Without loosing generality, we will assume that the precondition is satisfiable (which can be effectively checked due to Theorem 2).

For the rest of this section, let us fix a set of array variables $\mathbf{a}=\left\{a_{1}, a_{2}, \ldots, a_{k}\right\}$ and a set of scalar variables $\mathbf{b}=\left\{b_{1}, b_{2}, \ldots, b_{m}\right\}$. As shown in [13], each $\exists^{*} \forall^{*}$-SIL formula can be equivalently written as a boolean combination of two kinds of formulae: 
(i) array properties of the form $\forall i . f \leq i \leq g \rightarrow v$, where $f$ and $g$ are bound terms, and $v$ is either: (1) $a_{p}[i] \sim B$, (2) $i-a_{p}[i] \sim n$, or (3) $a_{p}[i]-a_{q}[i+1] \sim n$, where $\sim \in\{\leq, \geq\}, 1 \leq p, q \leq k, n \in \mathbb{Z}$, and $B$ is a bound term.

(ii) Presburger constraints on scalar variables $\mathbf{b}$.

Let us now fix a (normalised) pre-condition formula $\varphi(\mathbf{a}, \mathbf{b})$ of $\exists^{*} \forall^{*}$-SIL. By pushing negation inwards (using DeMorgan's laws) and eliminating it from Presburger constraints on scalar variables, we obtain a boolean combination of formulae of the forms (i) or (ii) above, where only array properties may occur negated.

W.l.o.g., we consider only pre-condition formulae without disjunctions 3 For such formulae $\varphi$, we build CA $A_{\varphi}$ with index counters $\mathbf{i}=\left\{i_{1}, i_{2}, \ldots, i_{k}\right\}$, value counters $\mathbf{x}=$ $\left\{x_{1}, x_{2}, \ldots, x_{k}\right\}$, and parameters $\mathbf{p}=\left\{p_{1}, p_{2}, \ldots, p_{m}\right\}$, corresponding to the scalars $\mathbf{b}$.

For a term or formula $f$, we denote by $\bar{f}$ the term or formula obtained from $f$ by replacing each $b_{q}$ by $p_{q}, 1 \leq q \leq m$, respectively. The construction of $A_{\varphi}$ is defined recursively on the structure of $\varphi$ :

- If $\varphi=\psi_{1} \wedge \psi_{2}$, then $A_{\varphi}=A_{\psi_{1}} \otimes A_{\psi_{2}}$.

- If $\varphi$ is a Presburger constraint on $\mathbf{b}$, then $A_{\varphi}=\left\langle X, Q,\left\{q_{i}\right\}, \rightarrow,\left\{q_{f}\right\}\right\rangle$ where:

- $X=\left\{p_{q} \mid b_{q} \in F V(\varphi) \cap B V a r, 1 \leq q \leq m\right\}$,

- $Q=\left\{q_{i}, q_{f}\right\}$,

- $q_{i} \stackrel{\bar{\varphi} \wedge \wedge_{x \in X} x^{\prime}=x}{\longrightarrow} q_{f}$ and $q_{f} \stackrel{\wedge_{x \in X} x^{\prime}=x}{\longrightarrow} q_{f}$.

- For $\varphi$ being $\forall i . f \leq i \leq g \rightarrow v, A_{\varphi}$ and $A_{\neg \varphi}$ have states $Q=\left\{q_{i}, q_{1}, q_{2}, q_{3}, q_{f}\right\}$, with $q_{i}$ and $q_{f}$ being the initial and final states, respectively. Intuitively, the automaton waits in $q_{1}$ increasing its index counters until the lower bound $f$ is reached, then moves to $q_{2}$ and checks the value constraint $v$ until the upper bound $g$ is reached. Finally, the control moves to $q_{3}$ and the automaton scans the rest of the array until the end. In each state, the automaton can also non-deterministically choose to idle, which is needed to ensure state-completeness when making a product of such CA. For $v$ of type (1) and (2), the automaton has one index $\left(i_{p}\right)$ and value $\left(x_{p}\right)$ counters, while for $v$ of type (3), there are two dependent index $\left(i_{p}, i_{q}\right)$ and value $\left(x_{p}, x_{q}\right)$ counters. The full definitions of $A_{\varphi}$ and $A_{\neg \varphi}$ are given in [4], for space reasons.

We aim now at computing the strongest dependency $\Delta\left(A_{\varphi}\right)$ between the index counters of $A_{\varphi}$, and, moreover, at showing that $A_{\varphi}$ is state-complete (cf. Definition 3). Since $A_{\varphi}$ is defined inductively, on the structure of $\varphi, \Delta\left(A_{\varphi}\right)$ can also be computed inductively. Let $\delta(\varphi)$ be the formula defined as follows:

- $\delta(\varphi)=\top$ if $\varphi$ is a Presburger constraint on $\mathbf{b}$,

- for $\varphi \equiv \forall i . f \leq i \leq g \rightarrow v, \delta(\varphi) \triangleq \delta(\neg \varphi) \triangleq \begin{cases}\top & \text { if } v \text { is } a_{p}[i] \sim B \text { or } i-a_{p}[i] \sim n, \\ i_{p}=i_{q} \text { if } v \text { is } a_{p}[i]-a_{q}[i+1] \sim n,\end{cases}$

$-\delta\left(\varphi_{1} \wedge \varphi_{2}\right)=\delta\left(\varphi_{1}\right) \wedge \delta\left(\varphi_{2}\right)$.

Theorem 3. Given a satisfiable $\exists^{*} \forall^{*}$-SIL formula $\varphi$, the following hold for the $C A$ $A_{\varphi}$ defined above: (1) $A_{\varphi}$ is state consistent, (2) $A_{\varphi}$ is state complete, (3) $A_{\varphi}$ and $\varphi$ correspond, and (4) $\delta\left(A_{\varphi}\right) \leftrightarrow \Delta\left(A_{\varphi}\right)$.

\footnotetext{
${ }^{3}$ Given a formula containing disjunctions, we put it in DNF and check each disjunct separately.
} 


\subsection{From Counter Automata to $\exists * \forall^{*}$-SIL}

The purpose of this section is to establish a dual connection, from counter automata to the $\exists^{*} \forall^{*}$ fragment of SIL. Since obviously, counter automata are much more expressive than $\exists^{*} \forall^{*}$-SIL, our first concern is to abstract a given state-consistent CA $A$ by a set of restricted $\mathrm{CA} \mathcal{A}_{1}^{K}, \mathcal{A}_{2}^{K}, \ldots, \mathcal{A}_{n}^{K}$, such that $\Sigma(A) \subseteq \bigcap_{i=1}^{n} \Sigma\left(\mathcal{A}_{i}^{K}\right)$, and for each $\mathcal{A}_{i}^{K}, 1 \leq$ $i \leq n$, to generate an $\exists^{*} \forall^{*}$-SIL formula $\varphi_{i}$ that corresponds to it. As a result, we obtain a formula $\varphi_{A}=\bigwedge_{i=1}^{n} \varphi_{i}$ such that $\left.\Sigma(A) \subseteq\left[\varphi_{A}\right]\right]$.

Let $\rho\left(X, X^{\prime}\right)$ be a relation on a given set of integer variables $X$, and $I(X)$ be a predicate defining a subset of $\mathbb{Z}^{k}$. We denote by $\rho(I)=\left\{X^{\prime} \mid \exists X \in I .\left\langle X, X^{\prime}\right\rangle \in R\right\}$ the image of $I$ via $R$, and we let $\rho \wedge I=\left\{\left\langle X, X^{\prime}\right\rangle \in \rho \mid X \in I\right\}$. By $\rho^{n}$, we denote the $n$-times relational composition $\rho \circ \rho \circ \ldots \circ \rho, \rho^{*}=\bigvee_{n>0} \rho^{n}$ is the reflexive and transitive closure of $\rho$, and $T$ is the entire domain $\mathbb{Z}^{k}$. If $\rho$ is a difference bound constraint, then $\rho^{n}$ is also a difference bound constraint, for a fixed constant $n>0$, and $\rho^{*}$ is a Presburger definable relation [7]5] (but not necessarily a difference bound constraint).

Let $\mathcal{D}(\rho)$ denote the strongest (in the logical sense) difference bound relation $D$ s.t. $\rho \subseteq D$. If $\rho$ is Presburger definable, $\mathcal{D}(\rho)$ can be effectively computed 4 , and, moreover, if $\rho$ is a finite union of $n$ difference bound relations, this takes $O\left(n \times 4 k^{2}\right)$ time 5 , where $k$ is the number of free variables in $\rho$.

We now define the restricted class of $\mathrm{CA}$, called flat counter automata with difference bound constraints (FCADBC) into which we abstract the given CA. A control path in a CA $A$ is a finite sequence $q_{1} q_{2} \ldots q_{n}$ of control states such that, for all $1 \leq i<n$, there exists a transition rule $q_{i} \stackrel{\varphi_{i}}{\longrightarrow} q_{i+1}$. A cycle is a control path starting and ending in the same control state. An elementary cycle is a cycle in which each state appears only once, except for the first one, which appears both at the beginning and at the end. A CA is said to be flat (FCA) iff each control state belongs to at most one elementary cycle. An FCA such that every relation labelling a transition occurring in an elementary cycle is a DBC, and the other relations are Presburger constraints, is called an FCADBC.

With these notations, we define the $K$-unfolding of a one-state self-loop CA $A_{\rho}=$ $\langle X,\{q\},\{q\}, q \stackrel{\rho}{\rightarrow} q,\{q\}\rangle$ as the FCADBC $A_{\rho}^{K}=\left\langle X, Q_{\rho}^{K},\left\{q_{1}\right\}, \rightarrow_{\rho}^{K}, Q_{\rho}^{K}\right\rangle$, where $Q_{\rho}^{K}=$ $\left\{q_{1}, q_{2}, \ldots, q_{K}\right\}$ and $\rightarrow{ }_{\rho}^{K}$ is defined such that $q_{i} \stackrel{\rho}{\rightarrow} q_{i+1}, 1 \leq i<K$, and $q_{K} \stackrel{\rho^{K}(\top) \wedge \rho}{\longrightarrow} q_{K}$. The $K$-abstraction of $A_{\rho}$, denoted $\mathcal{A}_{\rho}^{K}$ (cf. Fig. 2), is obtained from $A_{\rho}^{K}$ by replacing the transition rule $q_{K} \stackrel{\rho^{K}(T) \wedge \rho}{\longrightarrow} q_{K}$ with the difference bound rule $q_{K} \stackrel{\mathcal{D}\left(\rho^{K}(\top) \wedge \rho\right)}{\longrightarrow} q_{K}$. Intuitively, the information gathered by unfolding the concrete relation $K$ times prior to the abstraction on the loop $q_{K} \rightarrow q_{K}$, allows to tighten the abstraction, according to the $K$ parameter. Notice that the $\mathcal{A}_{\rho}^{K}$ abstraction of a relation $\rho$ is an FCADBC with exactly one initial state, one self-loop, and all states final. The following lemma proves that the abstraction is sound, and that it can be refined, by increasing $K$.

Lemma 1. Given a relation $\rho\left(X, X^{\prime}\right)$ on $X=\left\{x_{1}, \ldots, x_{k}\right\}$, the following facts hold: (1) $\operatorname{Tr}\left(A_{\rho}\right)=\operatorname{Tr}\left(A_{\rho}^{K}\right) \subseteq \operatorname{Tr}\left(\mathcal{A}_{\rho}^{K}\right)$, for all $K>0$, and (2) $\operatorname{Tr}\left(\mathcal{A}_{\rho}^{K_{2}}\right) \subseteq \operatorname{Tr}\left(\mathcal{A}_{\rho}^{K_{1}}\right)$ if $K_{1} \leq K_{2}$.

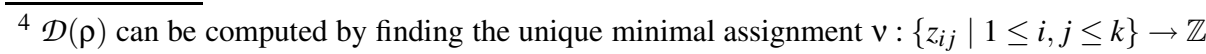
that satisfies the Presburger formula $\phi(\mathbf{z}): \forall X \forall X^{\prime} . \rho\left(X, X^{\prime}\right) \rightarrow \bigwedge_{x_{i}, x_{j} \in X \cup X^{\prime}} x_{i}-x_{j} \leq z_{i j}$.

${ }^{5}$ If $\rho=\rho_{1} \vee \rho_{2} \vee \ldots \vee \rho_{n}$, and each $\rho_{i}$ is represented by a $(2 k)^{2}$-matrix $M_{i}, \mathcal{D}(\rho)$ is given by the pointwise maximum among all matrices $M_{i}, 1 \leq i \leq n$. 

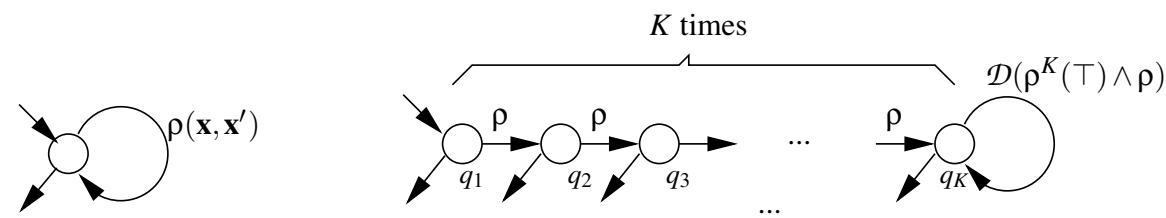

Fig. 2. $K$-abstraction of a relation

For the rest of this section, assume a set of arrays $\mathbf{a}=\left\{a_{1}, a_{2}, \ldots, a_{k}\right\}$ and a set of scalars $\mathbf{b}=\left\{b_{1}, b_{2}, \ldots, b_{m}\right\}$. At this point, we can describe an abstraction for counter automata that yields from an arbitrary state-consistent CA $A$, a set of state-consistent FCADBC $\mathcal{A}_{1}^{K}, \mathcal{A}_{2}^{K}, \ldots, \mathcal{A}_{n}^{K}$, whose intersection of sets of recognised states is a superset of the original one, i.e., $\Sigma(A) \subseteq \bigcap_{i=1}^{n} \Sigma\left(\mathcal{A}_{i}^{K}\right)$. Let $A$ be a state-consistent CA with counters $X$ partitioned into value counters $\mathbf{x}=\left\{x_{1}, \ldots, x_{k}\right\}$, index counters $\mathbf{i}=\left\{i_{1}, \ldots, i_{k}\right\}$, parameters $\mathbf{p}=\left\{p_{1}, \ldots, p_{m}\right\}$ and working counters $\mathbf{w}$. We assume that the only actions on an index counter $i \in \mathbf{i}$ are tick $\left(i^{\prime}=i+1\right)$ and idle $\left(i^{\prime}=i\right)$, which is sufficient for the CA that we generate from SIL or loops.

The main idea behind the abstraction method is to keep the idle relations separate from ticks. Notice that, by combining (i.e., taking the union of) idle and tick transitions, we obtain non-deterministic relations (w.r.t. index counters) that may break the stateconsistency requirement imposed on the abstract counter automata. Hence, the first step is to eliminate the idle transitions.

Let $\delta$ be an over-approximation of the dependency $\Delta(A)$, i.e., $\Delta(A) \rightarrow \delta$. In particular, if $A$ was obtained as in Theorem 1 by composing a pre-condition automaton with a transducer $T$, and if we dispose of an over-approximation $\delta$ of $\Delta(T)$, i.e., $\Delta(T) \rightarrow \delta$, we have that $\Delta(A) \rightarrow \delta$, cf. Theorem 1 -any over-approximation of the transducer's dependency is an over-approximation of the dependency for the post-image CA.

The dependency $\delta$ induces an equivalence relation on index counters: for all $i, j \in \mathbf{i}$, $i \simeq_{\delta} j$ iff $\delta \rightarrow i=j$. This relation partitions $\mathbf{i}$ into $n$ equivalence classes $\left[i_{s_{1}}\right],\left[i_{s_{2}}\right], \ldots,\left[i_{s_{n}}\right]$, where $1 \leq s_{1}, s_{2}, \ldots, s_{n} \leq k$. Let us consider $n$ identical copies of $A: A_{1}, A_{2}, \ldots, A_{n}$. Each copy $A_{j}$ will be abstracted w.r.t. the corresponding $\simeq_{\delta}$-equivalence class $\left[i_{s_{j}}\right]$ into $\mathcal{A}_{j}^{K}$ obtained as in Fig. 2. Thus we obtain $\Sigma(A) \subseteq \bigcap_{j=1}^{n} \Sigma\left(\mathcal{A}_{j}^{K}\right)$, by Lemma1.

We describe now the abstraction of the $A_{j}$ copy of $A$ into $\mathcal{A}_{j}^{K}$. W.1.o.g., we assume that the control flow graph of $A_{j}$ consists of one strongly connected component (SCC) otherwise we separately replace each (non-trivial) SCC by a flat CA obtained as described below. Out of the set of relations $\mathcal{R}_{A_{j}}$ that label transitions of $A_{j}$, let $v_{1}^{j}, \ldots, v_{p}^{j}$ be the set of $i d l e$ relations w.r.t. $\left[i_{s_{j}}\right]$, i.e., $v_{t}^{j} \rightarrow \bigwedge_{i \in\left[i_{s_{j}}\right]} i^{\prime}=i, 1 \leq t \leq p$, and $\theta_{1}^{j}, \ldots, \theta_{q}^{j}$ be the set of tick relations w.r.t. $\left[i_{s_{j}}\right]$, i.e., $\theta_{t}^{j} \rightarrow \bigwedge_{i \in\left[i_{s_{j}}\right]} i^{\prime}=i+1,1 \leq t \leq q$. Note that since we consider index counters belonging to the same $\simeq_{\delta}$-equivalence class, they either all idle or all tick, hence $\left\{v_{1}^{j}, \ldots, v_{p}^{j}\right\}$ and $\left\{\theta_{1}^{j}, \ldots, \theta_{q}^{j}\right\}$ form a partition of $\mathcal{R}_{A_{j}}$.

Let $\Upsilon_{j}=\mathcal{D}\left(\bigvee_{t=1}^{p} v_{t}^{j}\right)$ be the best difference bound relation that approximates the idle part of $A_{j}$, and $\Upsilon_{j}^{*}$ be its reflexive and transitive closure 6 . Let $\Theta_{j}=\bigvee_{t=1}^{q} \mathcal{D}\left(\Upsilon_{j}^{*}\right) \circ \theta_{t}^{j}$, and

\footnotetext{
${ }^{6}$ Since $\Upsilon_{j}$ is a difference bound relation, by [7|5], we have that $\Upsilon_{j}^{*}$ is Presburger definable.
} 
let $A_{\Theta_{j}}$ be the one-state self-loop automaton whose transition is labelled by $\Theta_{j}$, and $\mathcal{A}_{j}^{K}$ be the $K$-abstraction of $A_{\Theta_{j}}$ (cf. Fig. 2). It is to be noticed that the abstraction replaces a state-consistent FCA with a single SCC by a set of state-consistent FCADBC with one self-loop. The soundness of the abstraction is proved in the following:

Lemma 2. Given a state-consistent $C A A$ with index counters $\mathbf{i}$ and a dependency $\delta$ s.t. $\Delta(A) \rightarrow \delta$, let $\left[i_{s_{1}}\right],\left[i_{s_{2}}\right], \ldots,\left[i_{s_{n}}\right]$ be the partition of $\mathbf{i}$ into $\simeq_{\delta}$-equivalence classes. Then each $\mathcal{A}_{i}^{K}, 1 \leq i \leq n$ is state-consistent, and $\Sigma(A) \subseteq \bigcap_{i=1}^{n} \Sigma\left(\mathcal{A}_{i}^{K}\right)$, for any $K \geq 0$.

The next step is to build, for each FCADBC $\mathcal{A}_{i}^{K}, 1 \leq i \leq n$, an $\exists^{*} \forall^{*}$-SIL formula $\varphi_{i}$ such that $\left.\Sigma\left(\mathcal{A}_{i}^{K}\right)=\left[\llbracket \varphi_{i}\right]\right]$, for all $1 \leq i \leq n$, and, finally, let $\varphi_{A}=\bigwedge_{i=1}^{n} \varphi_{i}$ be the needed formula. The generation of the formulae builds on that we are dealing with CA of the form depicted in the right of Fig. 27

For a relation $\varphi\left(X, X^{\prime}\right), X=\mathbf{x} \cup \mathbf{p}$, let $\mathcal{T}_{i}(\varphi)$ be the SIL formula obtained by replacing (1) each unprimed value counter $x_{s} \in F V(\varphi) \cap \mathbf{x}$ by $a_{s}[i]$, (2) each primed value counter $x_{s}^{\prime} \in F V(\varphi) \cap \mathbf{x}^{\prime}$ by $a_{s}[i+1]$, and (3) each parameter $p_{r} \in F V(\varphi) \cap \mathbf{p}$ by $b_{r}$, for $1 \leq s \leq k$, $1 \leq r \leq m$.

For the rest, fix an automaton $\mathcal{A}_{j}^{K}$ of the form from Fig. 2 for some $1 \leq j \leq n$, and let $q_{p} \stackrel{\rho}{\rightarrow} q_{p+1}, 1 \leq p<K$, be its sequential part, and $q_{K} \stackrel{\lambda}{\rightarrow} q_{K}$ its self-loop. Let $\left[i_{s_{j}}\right]=\left\{i_{t_{1}}, i_{t_{2}}, \ldots, i_{t_{q}}\right\}$ be the set of relevant index counters for $\mathcal{A}_{j}^{K}$, and let $\mathbf{x}_{r}=$ $\mathbf{x} \backslash\left\{x_{t_{1}}, \ldots, x_{t_{q}}\right\}$ be the set of redundant value counters. With these notations, the desired formula is defined as $\varphi_{j}=\left(\bigvee_{l=1}^{K-1} \tau(l)\right) \vee(\exists b . b \geq 0 \wedge \tau(K) \wedge \omega(b))$, where:

$$
\begin{aligned}
& \tau(l): \bigwedge_{s=0}^{l-1} \mathcal{I}_{s}\left(\exists \mathbf{i}, \mathbf{x}_{r}, \mathbf{x}_{r}^{\prime}, \mathbf{w} . \rho\right) \\
& \omega(b):\left(\forall i . K \leq \mathfrak{j}<K+b \rightarrow \mathcal{T}_{i}\left(\exists \mathbf{i}, \mathbf{x}_{r}, \mathbf{x}_{r}^{\prime}, \mathbf{w} . \lambda\right)\right) \wedge \\
& \mathcal{T}_{0}\left(\exists \mathbf{i}, \mathbf{x}, \mathbf{x}^{\prime}, \mathbf{w} . \lambda^{b}\left[K / i_{t_{1}}, \ldots, i_{t_{q}}\right]\left[K+b-1 / i_{t_{1}}^{\prime}, \ldots, i_{t_{q}}^{\prime}\right]\right)
\end{aligned}
$$

Here, $b \in B$ Var is a fresh scalar denoting the number of times the self-loop $q_{K} \stackrel{\lambda}{\rightarrow} q_{K}$ is iterated. $\lambda^{b}$ denotes the formula defining the $b$-times composition of $\lambda$ with itself 8

Intuitively, $\tau(l)$ describes arrays corresponding to runs of $\mathcal{A}_{j}^{K}$ from $q_{1}$ to $q_{l}$, for some $1 \leq l \leq K$, without iterating the self-loop $q_{K} \stackrel{\lambda}{\rightarrow} q_{K}$, while $\omega(b)$ describes the arrays corresponding to runs going through the self-loop $b$ times. The second conjunct of $\omega(b)$ uses the closed form of the $b$-th iteration of $\lambda$, denoted $\lambda^{b}$, in order to capture the possible relations between $b$ and the scalar variables $\mathbf{b}$ corresponding to the parameters $\mathbf{p}$ in $\lambda$, created by iterating the self-loop.

Theorem 4. Given a state-consistent $C A A$ with index counters $\mathbf{i}$ and given a dependency $\delta$ such that $\Delta(A) \rightarrow \delta$, we have $\Sigma(A) \subseteq\left[\left[\varphi_{A}\right]\right]$, where:

- $\varphi_{A}=\bigwedge_{i=1}^{n} \varphi_{i}$, where $\varphi_{i}$ is the formula corresponding to $\mathcal{A}_{i}^{K}$, for all $1 \leq i \leq n$, and

- $\mathcal{A}_{1}^{K}, \mathcal{A}_{2}^{K}, \ldots, \mathcal{A}_{n}^{K}$ are the $K$-abstractions corresponding to the equivalence classes induced by $\delta$ on $\mathbf{i}$.

\footnotetext{
${ }^{7}$ In case we start from a CA with more SCCs, we get a CA with a DAG-shaped control flow interconnecting components of the form depicted in Fig. 2 after the abstraction. Such a CA may be converted to SIL by describing each component by a formula as above, parameterised by its beginning and final index values, and then connecting such formulae by conjunctions within particular control branches and taking a disjunction of the formulae derived for the particular branches. Due to lack of space, we give this construction in detail in [4] only.

${ }^{8}$ Since $\lambda$ is difference bound relation, $\lambda^{b}$ can be defined by a Presburger formula [7]5].
} 


\section{Array Manipulating Programs}

We consider programs consisting of assignments, conditional statements, and non-nested while loops in the form shown in Fig. 4 working over arrays AVar and scalar variables $B V a r$ (for a formal syntax, see [4]). In a loop, we assume a 1:1 correspondence between the set of arrays AVar and the set of indices IVar. In other words, each array is associated exactly $b \in$ BVar,$a \in$ AVar,$i \in I$ Var $, n \in \mathbb{Z}, c \in \mathbb{N}$

$$
\begin{aligned}
& A S G N::=L H S=R H S \\
& L H S \quad:=b \mid a[i+c] \\
& T R M \quad:=L H S \mid i \\
& R H S \quad:=T R M|-T R M| T R M+n \\
& C N D \quad:=C N D \& \& C N D \mid R H S \leq R H S
\end{aligned}
$$

Fig. 3. Assignments and conditions one index variable. Each index $i \in I V a r$ is initialised at the beginning of a loop using an expression of the form $b+n$ where $b \in B$ Var and $n \in \mathbb{Z}$. The indices are local to the loop. The body $S_{1}^{l} ; \ldots ; S_{n}^{l}$; of each loop branch consists of zero or more assignments followed by a single index increment statement incr $(I), I \subseteq$ IVar. The syntax of the assignments and boolean expressions used in conditional statements is shown in Fig. 3 We consider a simple syntax to make the presentation of the proposed techniques easier: various more complex features can be handled by straightforwardly extending the techniques described below.

A state of a program is a pair $\langle l, s\rangle$ where $l$ is a line of the program and $s$ is a state $\langle\alpha, \imath\rangle$ defined as in Section 3 . The semantics of program statements is the usual one (e.g., [19]). For simplicity of the further constructions, we assume that no out-of-bound array references occur in the programs - such situations are considered in [4].

Considering the program statements given in Fig. 3, we have developed a strongest post-condition calculus for the $\exists^{*} \forall^{*}$-SIL fragment. This calculus captures the semantics of the assignments and conditionals, and is used to deal with the sequential parts of the program (the blocks of statements outside the loops). It is also shown that $\exists^{*} \forall^{*}$-SIL is closed for strongest post-conditions. Full details are given in [4].

\subsection{From Loops to Counter Automata}

Given a loop $L$ starting at control line $l$, such that $l^{\prime}$ is the control line immediately following $L$, we denote by $\Theta_{L}=\left\{\langle s, t\rangle \mid\right.$ there is a run of $L$ from $\langle l, s\rangle$ to $\left.\left\langle l^{\prime}, t\right\rangle\right\}$ the transition relation induced by $L Q$ We define the loop dependency $\delta_{L}$ as the conjunction of equalities $i_{p}=i_{q}, i_{p}, i_{q} \in$ IVar, where (1) $e_{p} \equiv e_{q}$ where $e_{1}$ and $e_{2}$ are the expressions initialising $i_{p}$ and $i_{q}$ and (2) for each branch of $L$ finished by an index increment statement $\operatorname{incr}(I), i_{p} \in I \Longleftrightarrow i_{q} \in I$. The equivalence relation $\simeq_{\delta_{L}}$ on index counters is defined as before: $i_{p} \simeq_{\delta_{L}} i_{q}$ iff $=\delta_{L} \rightarrow i_{p}=i_{q}$.

Assume that we are given a loop $L$ as in Fig. (4) with AVar $=\left\{a_{1}, \ldots, a_{k}\right\}$, IVar $=\left\{i_{1}, \ldots, i_{k}\right\}$, and

$$
\begin{aligned}
& \text { while }_{a_{1}: i_{1}}=e_{1}, \ldots, a_{k}: i_{k}=e_{k} \\
& \quad \text { if }(C) \\
& \quad \text { else if }\left(C_{1}\right) S_{1}^{1} ; \ldots ; S_{n_{1}}^{1} ; \\
& \quad \text {.. } \\
& \quad \text { else if }\left(C_{h-1}^{2} ; \ldots ; S_{n_{2}}^{2} ;\right. \\
& \quad \text { else } S_{1}^{h-1} ; \ldots ; S_{n_{h}}^{h-1} ;
\end{aligned}
$$

Fig. 4. A while loop $B V a r=\left\{b_{1}, \ldots, b_{m}\right\}$ being the sets of array, index,

\footnotetext{
${ }^{9}$ Note that we ignore non-terminating runs of the loop in case there are some-our concern is not to check termination of the loop, but correctness of terminating runs of the loop.
} 
and scalar variables, respectively. Let $I_{1}, I_{2}, \ldots, I_{n} \subseteq$ IVar be the partition of IVar into equivalence classes, induced by $\simeq_{\delta_{L}}$. For $E$ being a condition, assignment, index increment, or an entire loop, we define $d_{E}: A \operatorname{Var} \rightarrow \mathbb{N} \cup\{\perp\}$ as $d_{E}(a)=\max \{c \mid$ $a[i+c]$ occurs in $E\}$ provided $a$ is used in $E$, and $d_{E}(a)=\perp$ otherwise. The transducer $T_{L}=\left\langle X, Q,\left\{q_{0}\right\}, \rightarrow,\left\{q_{\text {fin }}\right\}\right\rangle$, corresponding to the program loop $L$, is defined below:

- $X=\left\{x_{r}^{i}, x_{r}^{o}, i_{r} \mid 1 \leq r \leq k\right\} \cup\left\{w_{r, l}^{i} \mid 1 \leq r \leq k, 1 \leq l \leq d_{L}\left(a_{r}\right)\right\} \cup\left\{w_{r, l}^{o} \mid 1 \leq r \leq k, 0 \leq\right.$ $\left.l \leq d_{L}\left(a_{r}\right)\right\} \cup\left\{p_{r}^{i}, p_{r}^{o}, w_{r} \mid 1 \leq r \leq m\right\} \cup\left\{w_{N}\right\}$ where $x_{r}^{i / o}, 1 \leq r \leq k$, are input/output array counters, $p_{r}^{i / o}, 1 \leq r \leq k$, are parameters storing input/output scalar values, and $w_{r}, 1 \leq r \leq m$, are working counters used for the manipulation of arrays and scalars ( $w_{N}$ stores the common length of arrays).

- $Q=\left\{q_{0}, q_{\text {pre }}, q_{\text {loop }}, q_{\text {suf }}, q_{\text {fin }}\right\} \cup\left\{q_{r}^{l} \mid 1 \leq l \leq h, 0 \leq r<n_{l}\right\}$.

- The transition rules of $T_{L}$ are the following. We assume an implicit constraint $x^{\prime}=x$ for each counter $x \in X$ such that $x^{\prime}$ does not appear explicitly:

- $q_{0} \stackrel{\varphi}{\rightarrow} q_{\text {pre }}, \varphi=\bigwedge_{1 \leq r \leq m}\left(w_{r}=p_{r}^{i}\right) \wedge w_{N}>0 \wedge \bigwedge_{1 \leq r \leq k}\left(i_{r}=0 \wedge x_{r}^{i}=w_{r, 0}^{o}\right) \wedge$ $\bigwedge_{\substack{1 \leq r \leq k \\ 1 \leq l \leq d_{L}\left(a_{r}\right)}}\left(w_{r, l}^{i}=w_{r, l}^{o}\right)$ (the counters are initialised).

- For each $\simeq_{\delta_{L}}$-equivalence class $I_{j}, 1 \leq j \leq n, q_{\text {pre }} \stackrel{\varphi}{\rightarrow} q_{\text {pre }}$ with $\varphi=\bigwedge_{1 \leq r \leq k}\left(i_{r}<\right.$ $\left.\xi\left(e_{r}\right)\right) \wedge \xi(\operatorname{incr}(I))\left(T_{L}\right.$ copies the initial parts of the arrays untouched by $\left.L\right)$.

- $q_{\text {pre }} \stackrel{\varphi}{\rightarrow} q_{\text {loop }}, \varphi=\bigwedge_{1 \leq r \leq k} i_{r}=\xi\left(e_{r}\right)\left(T_{L}\right.$ starts simulating $\left.L\right)$.

- For each $1 \leq l \leq h, q_{\text {loop }} \stackrel{\varphi}{\rightarrow} q_{0}^{l}, \varphi=\xi(C) \wedge \wedge_{1 \leq r<l}\left(\neg \xi\left(C_{r}\right)\right) \wedge \xi\left(C_{l}\right)$ where $C_{h}=\top\left(T_{L}\right.$ chooses the loop branch to be simulated).

- For each $1 \leq l \leq h, 1 \leq r \leq n_{l}, q_{r-1}^{l} \stackrel{\xi\left(S_{r}^{l}\right)}{\longrightarrow} q$ where $q=q_{r}^{l}$ if $r<n_{l}$, and $q=q_{\text {loop }}$ otherwise (the automaton simulates one branch of the loop).

- $q_{\text {loop }} \stackrel{\varphi}{\rightarrow} q_{\text {suf }}, \varphi=\neg \xi(C) \wedge \bigwedge_{1 \leq r \leq m}\left(w_{r}=p_{r}^{o}\right)\left(T_{L}\right.$ finished the simulation of the actual execution of $L$ ).

- For each $\simeq_{\delta_{L}}$-equivalence class $I_{j}, 1 \leq j \leq n$, and $i_{r} \in I_{j}, q_{s u f} \stackrel{\varphi}{\rightarrow} q_{s u f}, \varphi=i_{r}<$ $w_{N} \wedge \xi\left(\operatorname{incr}\left(I_{j}\right)\right)$ (copy the array suffixes untouched by the loop).

- $q_{\text {suf }} \stackrel{\varphi}{\rightarrow} q_{\text {fin }}, \varphi=\bigwedge_{1 \leq r \leq k} i_{r}=w_{N}$ (all arrays are entirely processed).

The syntactical transformation $\xi$ of assignments and conditions preserves the structure of these expressions, but replaces each $b_{r}$ by the counter $w_{r}$ and each $a_{r}\left[i_{r}+c\right]$ by $w_{r, c}^{o}$ for $b_{r} \in B \operatorname{Var}, a_{r} \in A \operatorname{Var}, i_{r} \in I V a r$, and $c \in \mathbb{N}$. On the left-hand sides of the assignments, future values of the counters are used (cf. [4]). For increment statements we define, for all $i_{r} \in$ IVar:

$$
\begin{aligned}
& -\xi\left(\operatorname{incr}\left(i_{r}\right)\right): x_{r}^{i^{\prime}}=w_{r, 1}^{i} \wedge \wedge_{1<l \leq d_{L}\left(a_{r}\right)} w_{r, l-1}^{i^{\prime}}=w_{r, l}^{i} \wedge x_{r}^{o \prime}=w_{r, 0}^{o} \wedge \\
& \\
& \bigwedge_{0<l \leq d_{L}\left(a_{r}\right)} w_{r, l-1}^{o \prime}=w_{r, l}^{o} \wedge w_{r, d_{L}\left(a_{r}\right)}^{i^{\prime}}=w_{r, d_{L}\left(a_{r}\right)}^{o^{\prime}} \wedge i_{r}^{\prime}=i_{r}+1, \text { if } d_{L}\left(a_{r}\right)>0, \\
& -\xi\left(\operatorname{incr}\left(i_{r}\right)\right): x_{r}^{i^{\prime}}=w_{r, 0}^{o} \wedge x_{r}^{o \prime}=w_{r, 0}^{o} \wedge i_{r}^{\prime}=i_{r}+1, \text { if } d_{L}\left(a_{r}\right)=0 .
\end{aligned}
$$

For the increment of a set of indices, we extend this definition pointwise.

The main idea of the construction is the following. $T_{L}$ preserves the exact sequences of operations done on arrays and scalars in $L$, but performs them on suitably chosen 
counters instead, exploiting the fact that the program always accesses the arrays through a bounded window only, which is moving from the left to right. The contents of this window is stored in the working counters. The values stored in these counters are shifted among the counters at each increment step. In particular, the initial value of an array cell $a_{r}[l]$ is stored in $w_{r, d_{L}\left(a_{r}\right)}^{o}$ for $d_{L}\left(a_{r}\right)>0$ (the case of $d_{L}\left(a_{r}\right)=0$ is just a bit simpler). This value can then be accessed and/or modified via $w_{r, q}^{o}$ where $q \in\left\{d_{L}\left(a_{r}\right), \ldots, 0\right\}$ in the iterations $l-d_{L}\left(a_{r}\right), \ldots, l$, respectively, due to copying $w_{r, q}^{o}$ into $w_{r, q-1}^{o}$ whenever simulating $\operatorname{incr}\left(i_{r}\right)$ for $q>0$. At the same time, the initial value of $a_{r}[l]$ is stored in $w_{r, d_{L}\left(a_{r}\right)}^{i}$, which is then copied into $w_{r, q}^{i}$ for $q \in\left\{d_{L}\left(a_{r}\right)-1, \ldots, 1\right\}$ and finally into $x_{r}^{i}$, which happens exactly when $i_{r}$ reaches the value $l$. Within the simulation of the next $\operatorname{incr}\left(i_{r}\right)$ statement, the final value of $a_{r}[l]$ appears in $x_{r}^{o}$, which is exactly in accordance with how a transducer expresses a change in a certain cell of an array (cf. Def. 2).

Note also that the value of the index counters $i_{r}$ is correctly initialised via evaluating the appropriate initialising expressions $e_{r}$, it is increased at the same positions of the runs in both the loop $L$ and the transducer $T_{L}$, and it is tested within the same conditions. Moreover, the construction takes care of appropriately processing the array cells which are accessed less than the maximum possible number of times (i.e., less than $\delta_{L}\left(a_{r}\right)+1$ times) by (1) "copying" from the input $x_{r}^{i}$ counters to the output $x_{r}^{o}$ counters the values of all the array cells skipped at the beginning of the array by the loop, (2) by appropriately setting the initial values of all the working array counters before simulating the first iteration of the loop, and (3) by finishing the pass through the entire array even when the simulated loop does not pass it entirely.

The scalar variables are handled in a correct way too: Their input value is recorded in the $p_{r}^{i}$ counters, this value is initially copied into the working counters $w_{r}$ which are modified throughout the run of the transducer by the same operations as the appropriate program variables, and, at the end, the transducer checks whether the $p_{r}^{o}$ counters contain the right output value of these variables.

Finally, as for what concerns the dependencies, note that all the arrays whose indices are dependent in the loop (meaning that these indices are advanced in exactly the same loop branches and are initialised in the same way) are processed at the same time in the initial and final steps of the transducers (when the transducer is in the control states $q_{\text {pre }}$ or $q_{\text {suf }}$ ). Within the control paths leading from $q_{\text {loop }}$ to $q_{\text {loop }}$, indices of such arrays are advanced at the same time as these paths directly correspond to the branches of the loop. Hence, the working counters of these arrays have always the same value, which is, however, not necessarily the case for the other arrays.

It is thus easy to see that we can formulate the correctness of the translation as captured by the following Theorem.

Theorem 5. Given a program loop L, the following hold: (1) $T_{L}$ is a transition-consistent transducer, (2) $\Theta(L)=\Theta\left(T_{L}\right)$, and (3) $\Delta\left(T_{L}\right) \rightarrow \delta_{L}$.

The last point of Theorem 5 ensures that $\delta_{L}$ is a safe over-approximation of the dependency between the index counters of $T_{L}$. This over-approximation is used in Theorem 1 to check whether the post-image of a pre-condition automaton $A$ can be effectively computed, by checking $\delta_{T} \rightarrow \Delta(A)$. In order to meet requirements of Theorem 1 , one can extend $T_{L}$ in a straightforward way to copy from the input to the output all the arrays and integer variables which appear in the program but not in $L$. 


\section{Examples}

In order to validate our approach, we have performed proof-of-concept experiments with several programs handling integer arrays. Table 1 reports the size of the derived post-image automata (i.e., the CA representing the set of states after the main program loop) in numbers of control states and counters. The automata were slightly optimised using simple, lightweight static techniques (eliminating useless counters, compacting sequences of idling transitions with the first tick transition, eliminating clearly infeasible transitions). The result sizes give a hint on the simplicity and compactness of the obtained automata. As our prototype implementation is not completed to date, we have performed several steps of the translation into counter automata and back manually. The details of the experiments are given in [4].

The init example is the classical initialisation of an array with zeros. The partition example copies the positive elements of an array $a$ into another array $b$, and the negative ones into $c$. The insert example inserts an element on its corresponding position in a sorted array. The rotate example takes an array and rotates it by

Table 1. Examples

\begin{tabular}{||l|c|c||}
\hline program & control states & counters \\
\hline \hline init & 4 & 8 \\
\hline partition & 4 & 24 \\
\hline insert & 7 & 19 \\
\hline rotate & 4 & 15 \\
\hline
\end{tabular}
one position to the left. For all examples from Table 1, a human-readable post-condition describing the expected effect of the program has been inferred by our method.

\section{Conclusion}

In this paper, we have developed a new method for the verification of programs with integer arrays based on a novel combination of logic and counter automata. We use a logic of integer arrays to express pre- and post-conditions of programs and their parts, and counter automata and transducers to represent the effect of loops and to decide entailments. We have successfully validated our method on a set of experiments. A full implementation of our technique, which will allow us to do more experiments, is currently under way. In the future, we are, e.g., planning to investigate possibilities of using more static analyses to further shrink the size of the generated automata, optimisations to be used when computing transitive closures needed within the translation from CA to SIL, adjusted for the typical scenarios that happen in our setting, etc.

\section{References}

1. Beyer, D., Henzinger, T.A., Majumdar, R., Rybalchenko, A.: Invariant Synthesis for Combined Theories. In: Cook, B., Podelski, A. (eds.) VMCAI 2007. LNCS, vol. 4349, pp. 378394. Springer, Heidelberg (2007)

2. Beyer, D., Henzinger, T.A., Majumdar, R., Rybalchenko, A.: Path Invariants. In: Proc. of PLDI 2007, ACM SIGPLAN (2007)

3. Bouajjani, A., Habermehl, P., Jurski, Y., Sighireanu, M.: Rewriting Systems with Data: A Framework for Reasoning about Systems with Unbounded Structures over Infinite Data Domains. In: Csuhaj-Varjú, E., Ésik, Z. (eds.) FCT 2007. LNCS, vol. 4639, pp. 1-22. Springer, Heidelberg (2007) 
4. Bozga, M., Habermehl, P., Iosif, R., Konečný, F., Vojnar, T.: Automatic Verification of Integer Array Programs. Technical Report TR-2009-2, Verimag, Grenoble, France (2009)

5. Bozga, M., Iosif, R., Lakhnech, Y.: Flat Parametric Counter Automata. In: Bugliesi, M., Preneel, B., Sassone, V., Wegener, I. (eds.) ICALP 2006. LNCS, vol. 4052, pp. 577-588. Springer, Heidelberg (2006)

6. Bradley, A.R., Manna, Z., Sipma, H.B.: What's Decidable About Arrays? In: Emerson, E.A., Namjoshi, K.S. (eds.) VMCAI 2006. LNCS, vol. 3855, pp. 427-442. Springer, Heidelberg (2006)

7. Comon, H., Jurski, Y.: Multiple Counters Automata, Safety Analysis and Presburger Arithmetic. In: Y. Vardi, M. (ed.) CAV 1998. LNCS, vol. 1427. Springer, Heidelberg (1998)

8. Flanagan, C., Qadeer, S.: Predicate Abstraction for Software Verification. In: Proc. of POPL 2002. ACM, New York (2002)

9. Ghilardi, S., Nicolini, E., Ranise, S., Zucchelli, D.: Decision Procedures for Extensions of the Theory of Arrays. Annals of Mathematics and Artificial Intelligence 50 (2007)

10. Ghilardi, S., Nicolini, E., Ranise, S., Zucchelli, D.: Towards SMT Model Checking of Arraybased Systems. In: Armando, A., Baumgartner, P., Dowek, G. (eds.) IJCAR 2008. LNCS, vol. 5195, pp. 67-82. Springer, Heidelberg (2008)

11. Gopan, D., Reps, T.W., Sagiv, S.: A Framework for Numeric Analysis of Array Operations. In: POPL 2005. ACM, New York (2005)

12. Gulwani, S., McCloskey, B., Tiwari, A.: Lifting Abstract Interpreters to Quantified Logical Domains. In: POPL 2008. ACM, New York (2008)

13. Habermehl, P., Iosif, R., Vojnar, T.: A Logic of Singly Indexed Arrays. In: Cervesato, I., Veith, H., Voronkov, A. (eds.) LPAR 2008. LNCS (LNAI), vol. 5330, pp. 558-573. Springer, Heidelberg (2008)

14. Habermehl, P., Iosif, R., Vojnar, T.: What Else is Decidable about Integer Arrays? In: Amadio, R. (ed.) FOSSACS 2008. LNCS, vol. 4962, pp. 474-489. Springer, Heidelberg (2008)

15. Halbwachs, N., Péron, M.: Discovering Properties about Arrays in Simple Programs. In: Proc. of PLDI 2008. ACM, New York (2008)

16. Jhala, R., McMillan, K.: Array Abstractions from Proofs. In: Damm, W., Hermanns, H. (eds.) CAV 2007. LNCS, vol. 4590, pp. 193-206. Springer, Heidelberg (2007)

17. Kovács, L., Voronkov, A.: Finding Loop Invariants for Programs over Arrays Using a Theorem Prover. In: Chechik, M., Wirsing, M. (eds.) FASE 2009. LNCS, vol. 5503, pp. 470-486. Springer, Heidelberg (2009)

18. Lahiri, S.K., Bryant, R.E.: Indexed Predicate Discovery for Unbounded System Verification. In: Alur, R., Peled, D.A. (eds.) CAV 2004. LNCS, vol. 3114, pp. 135-147. Springer, Heidelberg (2004)

19. Manna, Z., Pnueli, A.: The Temporal Logic of Reactive and Concurrent Systems. Springer, Heidelberg (1992)

20. McMillan, K.: Quantified Invariant Generation Using an Interpolating Saturation Prover. In: Ramakrishnan, C.R., Rehof, J. (eds.) TACAS 2008. LNCS, vol. 4963, pp. 413-427. Springer, Heidelberg (2008)

21. Stump, A., Barrett, C.W., Dill, D.L., Levitt, J.R.: A Decision Procedure for an Extensional Theory of Arrays. In: Proc. of LICS 2001. IEEE Computer Society, Los Alamitos (2001) 